\title{
"Nurses and Midwives: clean care is in your hands": The 5th May 2020 World Health Organization SAVE LIVES: Clean Your Hands campaign
}

\author{
Alexandra Peters ${ }^{1}$, \\ Nasim Lotfinejad ${ }^{2}$, Chloé Guitart ${ }^{1}$, Alice Simniceanu ${ }^{3}$, \\ Maria Clara Padoveze ${ }^{3}$, Tcheun Borzykowski ${ }^{1}$, Benedetta Allegranzi ${ }^{3}$, Didier Pittet ${ }^{1}$ \\ ${ }^{1}$ Infection Control Program, University of Geneva Hospitals and Faculty of Medicine \\ 2Department of Research, Faculty of Medicine, Mashhad University of Medical Sciences, Mashhad, Iran \\ ${ }^{3}$ Infection Prevention and Control Global Unit, Department of Service Delivery and Safety, \\ World Health Organization, Geneva, Switzerland
}

10.3396/ijic.v16i1.002.20

Keywords: infection prevention and control, infection control, hand hygiene, nurses, midwives, World Health Organization, healthcare-associated infection

In honor of Florence Nightingale's 200 th birthday, the World Health Organization (WHO) has declared 2020 the "Year of the Nurse and Midwife". In addition to championing the nursing profession, Nightingale's role was also fundamental for the recognition of the importance of infection prevention and control (IPC), as she was among the first to recognize that a caregiver could transmit germs, and thus cause patient harm. Nurses and midwives make up nearly $50 \%$ of the global health workforce, ${ }^{1}$ and are the group of healthcare workers that have the most frequent contact with patients. This makes them pivotal figures in the fight against healthcare-associated infections (HAl) as well as neonatal and maternal sepsis.

On the $5^{\text {th }}$ May 2020, for the annual celebration of the SAVE LIVES: Clean Your Hands campaign, WHO will focus on the essential role that nurses and midwives play in contributing to saving millions of lives each year by championing clean care. Despite

\footnotetext{
Corresponding Author

Didier Pittet

Infection Control Program, University of Geneva Hospitals and Faculty of Medicine

E-mail:Didier.Pittet@hcuge.ch
} 
many improvements around the world, rates of HAI remain unacceptably high, and the majority of them are transmitted by healthcare workers' hands. Therefore, hand hygiene promotion strategies must be constantly reinforced and improved. Clean healthcare has recently been recognized by WHO as one of the most urgent challenges to be tackled by the global community over the next ten years. ${ }^{2}$ Actively engaging the expertise of nurses and midwives in the development, implementation and evaluation of hand hygiene promotion contributes to clean healthcare.

Along with recognizing the critical importance of nurses and midwives to patient care, the aim of the "Year of the Nurse and Midwife" is also to highlight that there is a major global shortage of healthcare workers, and that more than half of the shortage is of nurses and midwives. ${ }^{1}$ WHO estimates that for countries to succeed in reaching the Sustainable Development Goal \# 3 on health and well-being, the world will need an additional 9 million nurses and midwives by the year 2030. ${ }^{1}$ It has been proven that investing in education and job creation in the health and social sectors will result in improved health outcomes, global health security, and economic growth. ${ }^{1}$ Having adequate healthcare worker staffing reduces the risk of $\mathrm{HAl}$ and antimicrobial resistance, and is thus recommended by $\mathrm{WHO}$ as a core component of effective IPC programmes. ${ }^{3}$

It is crucial to recognize both the work and the immense responsibility that nurses and midwives carry: we cannot achieve Universal Health Coverage without investing in them. Everyone, including policy makers, healthcare workers, and patients themselves, can contribute to improving hand hygiene and preventing infections (Table I).

Please join us in celebrating this vital and often underappreciated group of HCW; "Nurses and Midwives: CLEAN CARE is in YOUR HANDS"!

The May 5, 2020, World Health Organization SAVE LIVES: Clean Your Hands campaign slogan and main promotional image (2020 hashtags: \#HandHygiene \#InfectionPrevention \#YONM \#SupportNursesAndMidwives \#Nurses2020 \#Midwives2020 \#HealthForAll). Campaign participants are invited to submit photos or selfies of them holding a board with the slogan and hashtags at www. CleanHandsSaveLives.org Notes

Table I. The 5 May 2020 World Health Organization SAVE LIVES: Clean Your Hands Campaign Calls to Action

\section{Campaign Participants}

\begin{tabular}{ll}
\hline Nurses & "Clean and safe care starts with you." \\
\hline Midwives & "Your hands make all the difference for mothers and babies." \\
\hline IPC leaders & "Empower nurses and midwives in providing clean care." \\
\hline Policy makers & "Increase nurse staffing levels to prevent infections and \\
& $\begin{array}{l}\text { improve quality of care. Create the means to empower nurses } \\
\text { and midwives." }\end{array}$ \\
\hline Patients and families & "Safer care for you, with you."
\end{tabular}

Abbreviations: IPC, infection prevention and control; WHO, World Health Organization. 


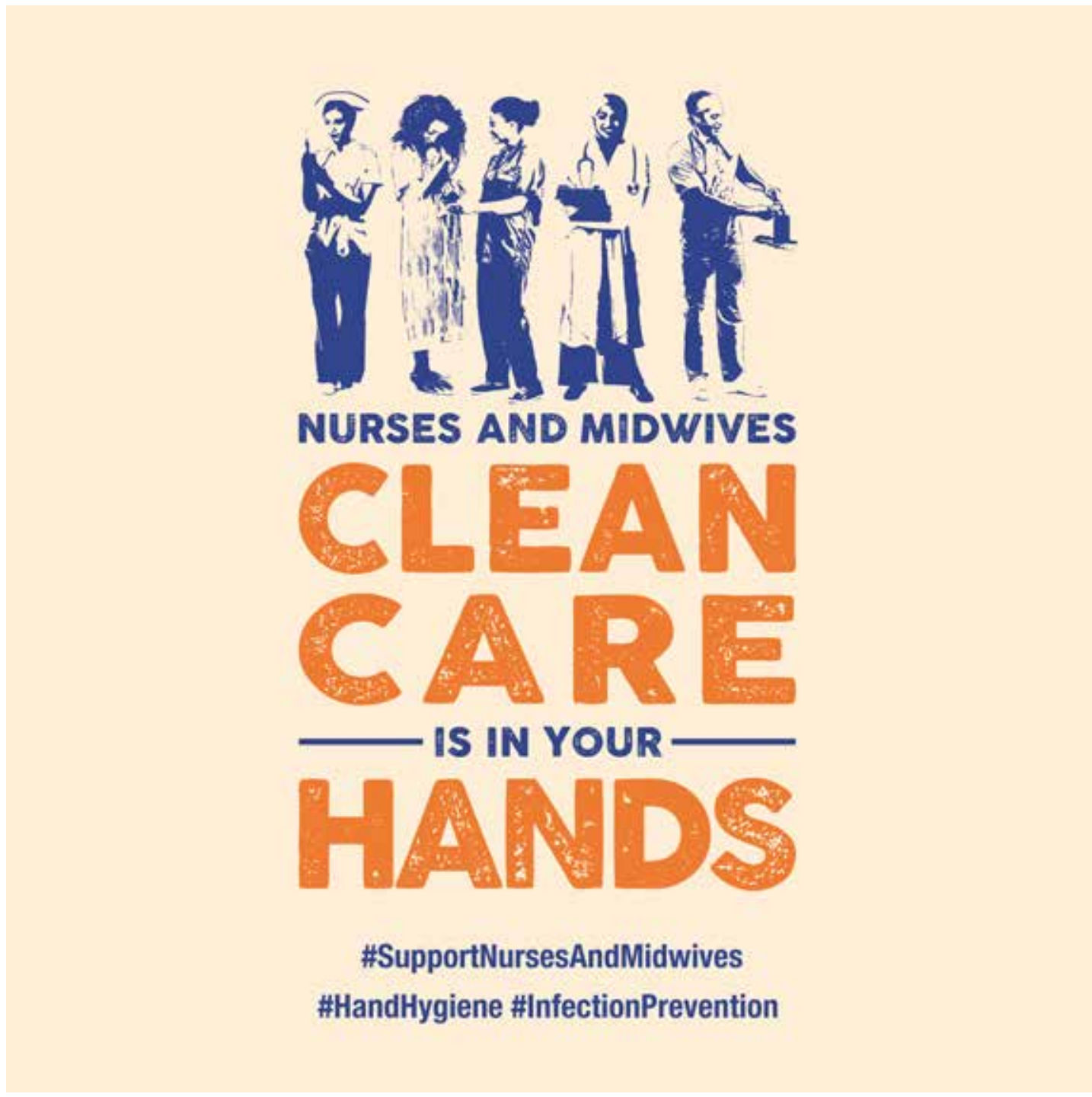

Figure May 5, 2020: "Nurses and Midwives, clean care is in your hands!"

\section{Disclaimer}

The views expressed in this article are those of the authors alone and do not necessarily represent the views, decisions, or policies of the institutions with which the authors are affiliated. The World Health Organization (WHO) takes no responsibility for the information provided or the views expressed in this article.

\section{Financial support}

This work is supported by WHO, Geneva, Switzerland, and the Infection Control Program, University of Geneva Hospitals and Faculty of Medicine, Geneva, Switzerland; hand hygiene research activities at the SPCI/WCC are also supported by the Swiss National Science Foundation (grant number 32003B_163262). Potential conflicts of interest.

\section{All authors}

No reported conflicts of interest. All authors have submitted the ICMJE Form for Disclosure of Potential Conflicts of Interest. Conflicts that the editors consider relevant to the content of the manuscript have been disclosed

\section{References}

1. "Nursing and midwifery". Fact sheets. World Health Organization. 9 January 2020. https://www.who.int/ news-room/fact-sheets/detail/nursing-and-midwifery

2. "Decade of Action" Geneva: World Health Organization. Accessed January 27, 2020. https://www.un.org/ sustainabledevelopment/decade-of-action/

3. "Guidelines on core components of infection prevention and control programmes at the national and acute health care facility level". Geneva: World Health Organization; 2016. https://www.who.int/gpsc/ipccomponents-guidelines/en/ 